\title{
Is cold gas fuelling the radio galaxy NGC 315?^
}

\author{
R. Morganti ${ }^{1,2}$, A. B. Peck ${ }^{3,4}$, T. A. Oosterloo ${ }^{1,2}$, G. van Moorsel $^{4}$, A. Capetti ${ }^{5}$, \\ R. Fanti ${ }^{6,7}$, P. Parma ${ }^{6}$, and H. R. de Ruiter ${ }^{6,8}$
}

1 Netherlands Foundation for Research in Astronomy, Postbus 2, 7990 AA, Dwingeloo, The Netherlands e-mail: morganti@astron.nl

2 Kapteyn Astronomical Institute, University of Groningen, P.O. Box 800, 9700 AV Groningen, The Netherlands

3 Joint ALMA Office, Av El Golf 40, piso 18, Santiago 7550108, Chile

${ }^{4}$ National Radio Astronomy Observatory, Socorro, NM 87801, USA

5 Osservatorio Astronomico di Torino, Strada Osservatorio 25, 10025 Pino Torinese, Italy

6 INAF, Istituto di Radioastronomia, Via Gobetti 101, 40129 Bologna, Italy

7 Dipartimento di Fisica dell’Università di Bologna, Via Irnerio 46, 40126 Bologna, Italy

8 Osservatorio Astronomico di Bologna, Via Ranzani, 1, 40127 Bologna, Italy

Received 31 May 2009 / Accepted 24 July 2009

\section{ABSTRACT}

\begin{abstract}
We present WSRT, VLA and VLBI observations of the H I absorption in the radio galaxy NGC 315 . The main result is that $t w o$ H I absorbing systems are detected against the central region. In addition to the known highly redshifted, very narrow component, we detect relatively broad (FWZI $\sim 150 \mathrm{~km} \mathrm{~s}^{-1}$ ) absorption. This broad component is redshifted by $\sim 80 \mathrm{~km} \mathrm{~s}^{-1}$ compared to the systemic velocity, while the narrow absorption is redshifted $\sim 490 \mathrm{~km} \mathrm{~s}^{-1}$. Both H I absorption components are spatially resolved at the pcscale of the VLBI observations. The broad component shows strong gradients in density (or excitation) and velocity along the jet. We conclude that this gas is physically close to the AGN, although the nature of the gas resulting in the broad absorption is not completely clear. The possibility that it is entrained by the radio jet (and partly responsible of the deceleration of the jet) appears unlikely. Gas located in a thick circumnuclear toroidal structure, with orientation similar to the dusty, circumnuclear disk observed with HST, cannot be completely ruled out although it appears difficult to reconcile with the observed morphology and kinematics of the H I. A perhaps more likely scenario is that the gas producing the broad absorption could be (directly or indirectly) connected with the fueling of the AGN, i.e. gas that is falling into the nucleus. If this is the case, the accretion rate derived is similar (considering all uncertainties) to that found for other X-ray luminous elliptical galaxies, although lower than that derived from the radio core luminosity for NGC 315. The data also show that, in contrast to the broad component, the density distribution of the narrow component is featureless. Moreover, in the WSRT observations we do detect a small amount of H I in emission a few kpc SW of the AGN, coincident with faint optical absorption features and at velocities very similar to the narrow absorption. This suggests that the gas causing the narrow absorption is not close to the AGN and is more likely caused by clouds falling into NGC 315 . The environment of NGC 315 turns out to be indeed quite gas rich since we detect five gas-rich companion galaxies in the immediate vicinity of NGC 315 .
\end{abstract}

Key words. Galaxies: active - Galaxies: individual: NGC 315 - radio lines: Galaxies

\section{Introduction}

\subsection{HI 21-cm absorption in low-luminosity radio galaxies}

The channeling of the gas to the very inner regions of a galaxy is considered to be the mechanism that can transform a "starving" black hole into an active nucleus. Mergers and interactions can play an important role in supplying the fuel and providing the conditions for the gas to reach the center (Wilson 1996). However, the relationship with mergers is not one-to-one and recent studies of radio galaxies have shown that the activity in some of these galaxies may be associated instead with the slow accretion of (hot) gas (Best et al. 2005, 2007; Croton et al. 2006). In the case of radio-loud AGN, the way the accretion of gas proceeds can have important implications in determining the characteristics of the radio source that is associated with the AGN.

* Based on observations with the Westerbork Synthesis Radio Telescope (WSRT), the Very Large Array (VLA) and Very Long Baseline Interferometer (VLBI).
In particular, the difference between powerful, edge-brightened and low-luminosity, edge-darkened radio galaxies could reflect a change in the mode of accretion: advective low efficiency/rate flow in the latter (Allen et al. 2006; Balmaverde et al. 2008) and standard optically thick accretion disks in the former. While the presence of thick tori predicted by unified schemes of AGN (see e.g. Antonucci 1993) is relatively well established for powerful radio galaxies through detections of obscuring atomic or molecular gas, X-ray absorption, or free-free absorption, the structure of the very inner regions of low-luminosity radio galaxies is still less defined. The study of obscuration toward the central regions of these galaxies (see e.g. Chiaberge et al. 1999; Chiaberge et al. 2002; Worrall et al. 2003; Balmaverde et al. 2006) has shown that the nuclear disks in these galaxies are geometrically and optically thin. This suggests that the standard pc-scale geometrically thick torus is not present in these low-luminosity radio galaxies.

On the other hand, the presence of gas in the nuclear regions can also affect the evolution of the radio sources (and in 
particular the radio jet) and the way the radio sources grow. Interaction between powerful radio jets and their environment is recognized to produce fast outflows that can be relevant in the evolution of the host galaxy (see e.g. Morganti et al. 2005; Holt et al. 2008). Entrainment of gas by the radio jet could also be crucial in slowing down the jet from relativistic to sub-relativistic velocities (see Laing et al. 2006).

Thus, it is clear that determining the gas distribution and kinematics in the nuclear regions is of paramount importance for understanding radio galaxies in general. Among the different gas components, the neutral hydrogen is particularly suited for this task. Atomic neutral hydrogen has been observed in absorption against the radio continuum in the central regions of a number of radio galaxies (see e.g. Heckman et al. 1983; Shostak et al. 1983; van Gorkom et al. 1990; Morganti et al. 2001; Vermeulen et al. 2003; Gupta et al. 2006). In some cases, though clearly not all, this H I absorption has been interpreted as due to gas distributed in circumnuclear disks/tori although there are surprisingly few examples where the spatially resolved signature of a rotating disk is actually observed. However, $\mathrm{H} \mathrm{I}$ absorption is not always found at the systemic velocity of the galaxy and, as mentioned above, more sensitive observations and detailed studies show that in many cases the kinematics of the gas can be disturbed and affected by the presence of the radio jets (Morganti et al. 2005 and references therein). Understanding the effect of the radio jets on this gas requires high spatial resolution observations that are at present quite scarce.

Here we present the results from our detailed study of $\mathrm{H} \mathrm{I}$ in the radio galaxy NGC 315. This includes Westerbork Synthesis Radio Telescope (WSRT), Very Large Array (VLA), and Global Very Long Baseline Interferometer (VLBI) observations. We have looked at this galaxy as part of a larger project to study, at both low and high resolution, the presence and the characteristics of the neutral hydrogen of a representative sample of low-luminosity radio galaxies (see Morganti 2002 for some details). The results of this statistical study will be presented in a forthcoming paper.

\subsection{Why NGC 315?}

NGC 315 is a bright elliptical galaxy. An accurate systemic velocity of $4942 \pm 6 \mathrm{~km} \mathrm{~s}^{-1}\left(z=0.01648^{1}\right)$ has been derived from stellar absorption lines by Trager et al. (2000). In the optical band, NGC 315 shows a highly inclined, very regular, circumnuclear disk, seen in absorption in the HST image (see Fig. 1). The dusty disk has a position angle of about $40^{\circ}$ and it extends to $r \sim 700 \mathrm{pc}$ with a mass of $\sim 1.9 \times 10^{7} M_{\odot}$ (Forbes 1991) calculated from the $S_{100 \mu \mathrm{m}}$ flux following Knapp et al. (1990). At its center there is an unresolved optical compact core (Capetti et al. 2000, 2002). A number of discrete optical absorption clouds, west of the nucleus at distances ranging from 3 to 7 arcsec, have been detected by Butcher et al. (1980). A mass limit of $10^{6} M_{\odot}$ has been derived for these clouds. The HST image shows additional absorption clouds to the SW. The dust disk is associated with a disk of ionized gas which appears to be in ordered rotation (Noel-Storr et al. 2003). CO emission was detected by Leon et al. (2003). The inferred mass of molecular hydrogen is $(3.0 \pm 0.3) \times 10^{8} M_{\odot}$.

NGC 315 hosts a giant $(\sim 1 \mathrm{Mpc})$ radio source that is also known as B2 0055+30 (Bridle et al. 1979; Mack et al. 1998;

\footnotetext{
1 Throughout this paper we use a Hubble constant $H_{\mathrm{o}}=70 \mathrm{~km} \mathrm{~s}^{-1}$ $\mathrm{Mpc}^{-1}$ and $\Omega_{\Lambda}=0.7$ and $\Omega_{\mathrm{M}}=0.3$. At the distance of NGC 315 this results in 1 arcsec $=0.335 \mathrm{kpc}$.
}
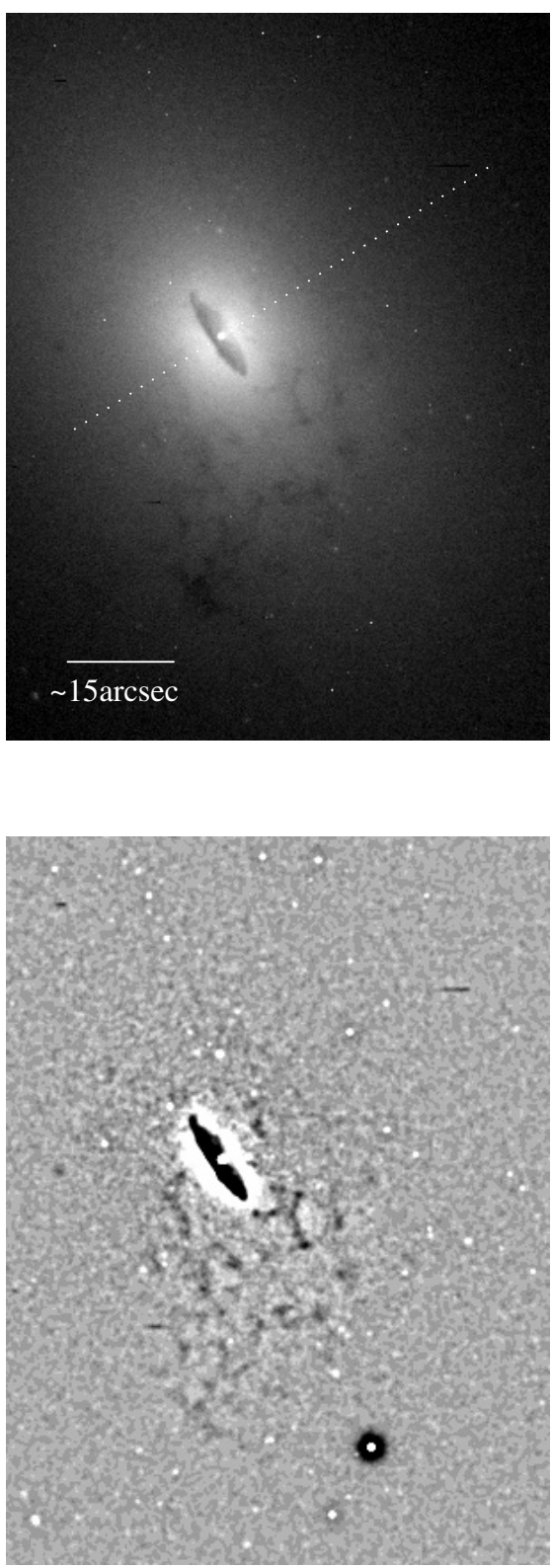

Fig. 1. Top: Image of NGC 315 obtained from HST (Capetti et al. 2000). The optical core is clearly visible as well as the dust disk and dust patches. The approximate direction of the radio jet is also indicated. Bottom: same image but highpass filtered and slightly smoothed afterward to highlight the dust patches around the nucleus.

Laing et al. 2006 and references therein). The source has a radio power of $\log P_{1.4 \mathrm{GHz}}=25.40 \mathrm{~W} \mathrm{~Hz}^{-1}$ and edge-darkened lobes (i.e. with Fanaroff-Riley type I structure, see Fig. 2). On VLBI scales, the source shows a core, a bright jet, and a faint counterjet (Cotton et al. 1999). The main jet is quite smooth. A multi-epoch study shows evidence for moving features, indicating an accelerating, mildly relativistic jet (Cotton et al. 1999). The jets show deceleration at larger distances and detailed modeling indicates that the angle of the jet to the line of sight is $\sim 38^{\circ}$ (Canvin et al. 2005).

The nuclear X-ray emission of NGC 315 is seen through a moderate intrinsic column density of $N_{\mathrm{H}} \sim 5 \times 10^{21} \mathrm{~cm}^{-2}$ (Worrall et al. 2003). Chandra typically finds column densities below $10^{22} \mathrm{~cm}^{-2}$ in FRI radio galaxy nuclei (e.g. Worrall et al. 2001), suggesting that the X-ray emission is associated with the sub-kpc-scale radio jets. 
Table 1. Instrumental parameters of the H I observations.

\begin{tabular}{lccc}
\hline \hline Field center $(\mathrm{J} 2000)$ & $\mathrm{RA}=0^{\mathrm{h}} 57^{\mathrm{m}} 48^{\mathrm{s}} ;$ Dec $=30^{\circ} 21^{\prime} 09^{\prime \prime}$ & \\
\hline Instrument & WSRT & VLA A-array & VLBI \\
Date of the observations & 30Jun00, 05Sep01 & $04 \mathrm{Dec} 00$ & $25 \mathrm{Nov01}$ \\
Integration time (h) & 11,10 & 0.5 & 4.5 \\
Synthesized beam $(\operatorname{arcsec})$ & $35 \times 18\left(-5^{\circ}\right)$ & $1.2 \times 1.0\left(26^{\circ}\right)$ & $9 \times 3$ mas $\left(-20^{\circ}\right)$ \\
Number of channels & 128 & 64 & 512 \\
Bandwidth $(\mathrm{MHz})$ & 10 & 6.5 & 8 \\
Central frequency $(\mathrm{MHz})$ & 1397.4 & 1397.4 & 1396.18 \\
Velocity resolution $\left(\mathrm{km} \mathrm{s}^{-1}\right)$ & 20 & 12 & 6.0 \\
rms noise in channel maps $\left(\mathrm{mJy} \mathrm{beam}^{-1}\right)$ & 0.21 & 0.86 & 0.7 \\
\hline
\end{tabular}

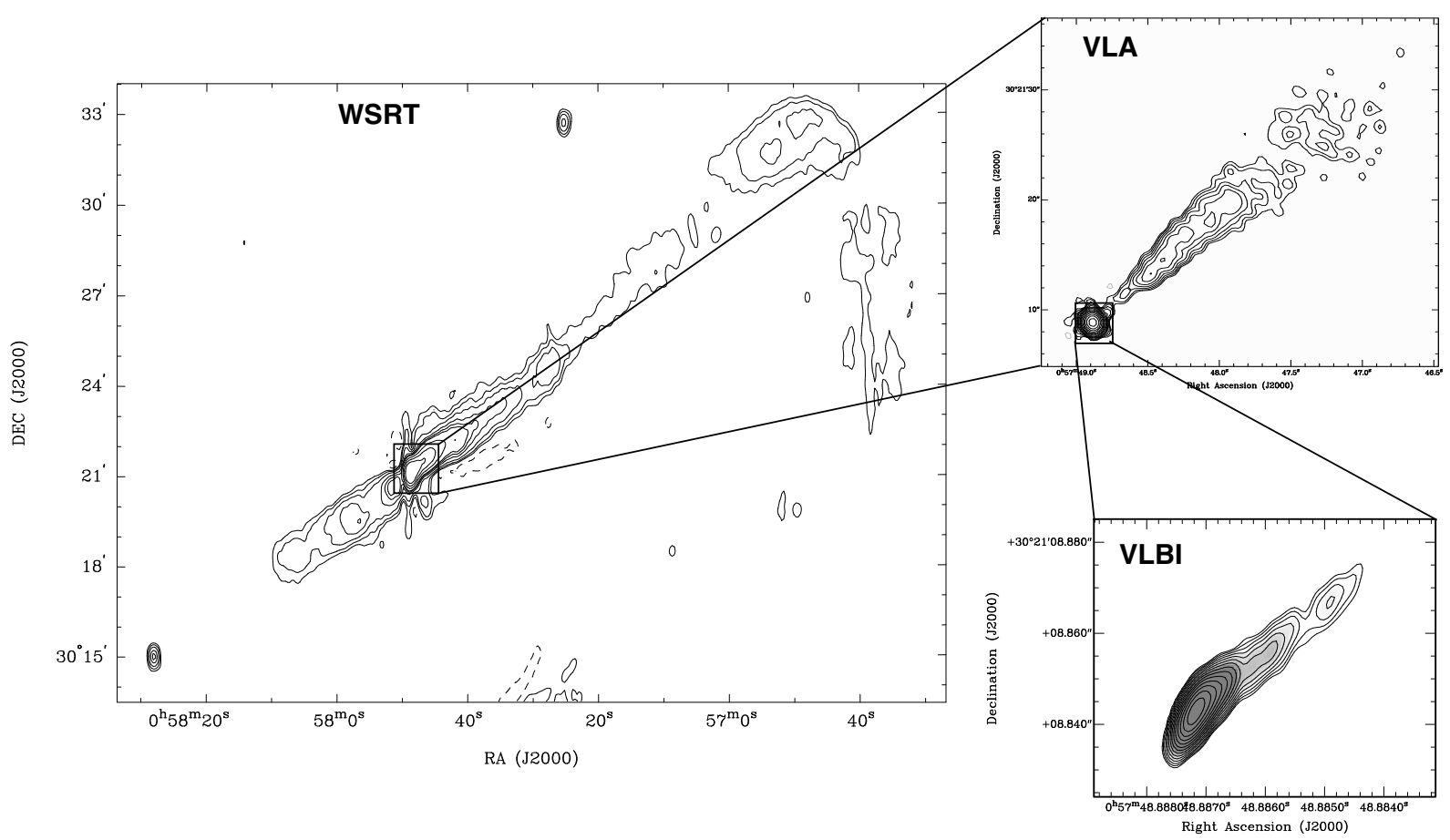

Fig. 2. Radio continuum image of NGC 315 at different angular resolutions (see Table 1): WSRT (left), VLA (middle) and VLBI (right). The images were obtained from the line-free channels, see text for details. Contour levels of the WSRT image $-1,1,2,4,8,12,16,32,64$ and $128 \mathrm{mJy}$ beam $^{-1}$.

NGC 315 has been observed in H I by different authors (Dressel et al. 1983; Heckman et al. 1983; Shostak et al. 1983; Chamaraux et al. 1987). In these studies, a narrow and highly redshifted ( $\sim 90 \mathrm{~km} \mathrm{~s}^{-1}$ with respect to the systemic velocity) $\mathrm{H}$ I absorption component has been detected. This H I absorption splits in two components when observed with high velocity resolution. These two components are very narrow (velocity width $\left.\sim 2.5 \mathrm{~km} \mathrm{~s}^{-1}\right)$ and very deep $(\tau=0.87$ and 0.21$)$ and separated by only $\sim 3 \mathrm{~km} \mathrm{~s}^{-1}$. A possible detection of an other broader $\mathrm{H}$ I absorption closer to the systemic velocity was reported by Heckman et al. (1983).

\section{WSRT and VLA observations and results}

The HI observations of NGC 315 reported in this paper have been carried out with various angular resolutions. The different observations have different purposes. In particular, we wanted to study in detail not only the $\mathrm{H}$ I absorption, but also investigate the possible presence of HI emission in and/or around this galaxy (Emonts et al., in prep.).

\subsection{Observations}

The low-resolution WSRT observations were done in order to investigate the presence of neutral hydrogen, both in emission and absorption. The relatively low spatial resolution of the WSRT is ideal when looking for low surface brightness and extended structures. The parameters of the observations are summarized in Table 1. 3C 147 was used as flux and bandpass calibrator. The data were calibrated and reduced using the MIRIAD package (Sault et al. 1995). The continuum subtraction was done using a linear fit through the line-free channels of each visibility record and subtracting this fit from all the frequency channels ("UVLIN"). The spectral-line cube was obtained using robust weighting set to 0.5 (Briggs 1995), i.e. intermediate between natural and uniform weighting. The data were Hanning smoothed to suppress the Gibbs ripples produced by the strong narrow absorption present in this galaxy. The resolution is $35 \times 18$ arcsec (p.a. $-5^{\circ}$ ) and the rms noise level is $\sim 0.21 \mathrm{mJy} \mathrm{beam}^{-1}$. In these observations we detected two $\mathrm{H}$ I absorption systems, as well as $\mathrm{H}$ I emission from NGC 315 and from five neighboring galaxies. 


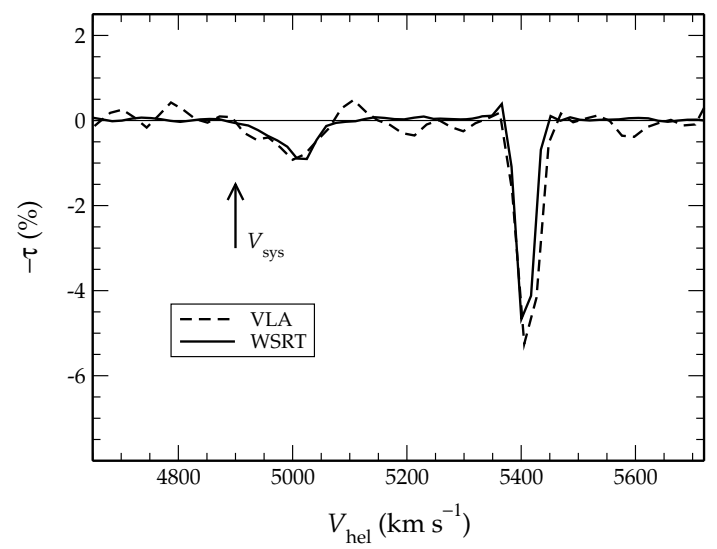

Fig. 3. H I profile (in optical depth) obtained from the WSRT (solid line) and the VLA (dashed) data. The two H I absorption systems are clearly visible. The systemic velocity from Trager et al. (2000) is also indicated. The differences in the profile, in particular for the narrow component, is likely due to the different velocity resolution of the two observations. The bump in the WSRT profile at velocities just below those of the narrow absorption, corresponds to the H I emission detected in NGC 315.

A continuum image was obtained from the line-free channels and is shown for reference in Fig. 2.

VLA observations of NGC 315 were obtained as part of a larger project aimed at studying the possible relation between the presence of $\mathrm{H}$ I absorption, the characteristics of the nuclear dust disks (seen by HST, Capetti et al. 2000) and the presence of optical cores (see Morganti et al. 2002 for preliminary results). The VLA observations have been carried out using the A-array to profit from the highest possible resolution with this telescope. The parameters are summarized in Table 1. The data reduction was done in a similar way as for the WSRT observations, again using the MIRIAD package. The continuum (from the line-free channels) and the line cube were produced with uniform weighting.

\subsection{The two $H \mathrm{l}$ absorption systems}

The first result of these observations is that two H I absorbing systems are detected in NGC 315. Apart from the very narrow and redshifted component (see Fig. 3), we also clearly detect the broad component that Heckman et al. (1983) reported as a probable detection. It is worth mentioning that there are only two other cases known of radio galaxies where two clearly separated H I absorbing systems have been observed: NGC 1275 (3C 84, see van Gorkom et al. 1989 and references therein) and 4C 31.04 (Mirabel 1990; Conway 1996).

The two components in NGC 315 are detected in all observations, also at the VLBI scale (see Sect. 6). Figure 3 shows the profiles of the two components. As already known (Dressel et al. 1983; Shostak et al. 1983), we find that the narrow H I absorption is highly redshifted (by $\sim 500 \mathrm{~km} \mathrm{~s}^{-1}$ ) compared to the systemic velocity. The narrow absorption is known to have a FWHM of only $2.5 \mathrm{~km} \mathrm{~s}^{-1}$ (Dressel et al. 1983). Note that, the observed width of the narrow, redshifted H I absorption is limited by the low velocity resolution (and Hanning smoothing) of our data, therefore appearing much larger than then $\sim 2.5 \mathrm{~km} \mathrm{~s}^{-1}$ detected with high velocity resolution by Arecibo (Dressel et al. 1983). Therefore, our low velocity resolution observations do not provide any new insight on this component. The integrated absorbed flux that we derive $\left(977 \mathrm{mJy} \mathrm{km} \mathrm{s}^{-1}\right)$ is also consistent with that obtained from Arecibo $\left(998 \mathrm{mJy} \mathrm{km} \mathrm{s}^{-1}\right.$ ).

The broad HI absorption, detected in both the WSRT and the VLA observations, has a full-width zero intensity (FWZI) in velocity of $\sim 150 \mathrm{~km} \mathrm{~s}^{-1}$ (FWHM $\sim 80 \mathrm{~km} \mathrm{~s}^{-1}$ ) and it is centered on $\sim 5020 \mathrm{~km} \mathrm{~s}^{-1}$. Although the broader component is located closer to the systemic velocity than the narrow component, it is nevertheless redshifted by about $80 \mathrm{~km} \mathrm{~s}^{-1}$. For this galaxy an accurate systemic velocity derived from stellar absorption lines is available $\left(V_{\text {sys }}=4942 \pm 6 \mathrm{~km} \mathrm{~s}^{-1}\right.$; Trager et al. 2000). This estimate of the systemic velocity is not affected by the disturbed kinematics that the ionized gas may have, thus the offset in velocity is significant.

The characteristics of both H I absorption components, when accounting for the different velocity resolution, are not different between the WSRT and the VLA data (see Fig. 3), indicating that the structures that produce the absorption have a size smaller than $\sim 1 \operatorname{arcsec}(0.5 \mathrm{kpc})$ and therefore the study of their morphology requires VLBI observations (see Sect. 6).

The peak of the continuum emission is $531 \mathrm{mJy}$ in the WSRT data, while the peak absorption of the broad component is $\sim-4.8 \mathrm{mJy}$ and the integrated absorbed flux that we derive is $\sim 353 \mathrm{mJy} \mathrm{km} \mathrm{s}^{-1}$. The resulting optical depth is $\sim 0.009$ and the corresponding column density of $2.5 \times 10^{18} T_{\text {spin }} / f \mathrm{~cm}^{-2}$ where $f$ is the covering factor. Similar values for the optical depth are derived from the VLA data.

\subsection{HI emission and the environment}

One of the aims of the low-resolution WSRT observations was to look for HI in emission in and around NGC 315. We do indeed find such emission in NGC 315 (Fig. 4 right), at the same location as the HST images show faint optical absorption features. The emission is detected at velocities from $V_{\text {hel }}=$ $5340 \mathrm{~km} \mathrm{~s}^{-1}$ up to that of the narrow absorption. The H I mass of the emission is $6.8 \times 10^{7} M_{\odot}$ and the peak column density is $1.0 \times 10^{20} \mathrm{~cm}^{-2}$. NGC 315 is, therefore, similar to other FRI radio galaxies (Morganti et al. 2009; Emonts et al. in preparation). Radio galaxies of this type do not appear to have large amounts of HI associated with the host galaxy (Emonts 2006; Emonts et al. 2006). Large amounts of neutral hydrogen have so far been detected only near compact radio galaxies (Emonts et al. 2006). In these sources, the gas is distributed in very extended disks/rings with large H I mass $\left(\gtrsim 10^{9} M_{\odot}\right)$, possibly indicating that a major merger has occurred in the not too distant past. This lack of such large, disk-like H I structures in extended FRI sources can be seen as another indication that triggering of the AGN does not happen through major mergers.

Apart from the emission in NGC 315, a number of neighboring galaxies are detected in HI. NGC 315 is part of the Zwicky cluster $0107.5+3212$ (Zwicky et al. 1961) which is located in the Perseus-Pisces filament. Garcia (1993) lists 18 galaxies as part of this group. We detect H I emission in 5 galaxies within a few hundred $\mathrm{km} \mathrm{s}^{-1}$ from the redshift of NGC 315 . The objects and their characteristics are listed in Table 2. All galaxies have an optical counterpart (two of them are 2MASS sources) and are not part of the list of Garcia (1993).

In Fig. 4, we give the total $\mathrm{H}$ I intensity image, showing the five companions. The environment of NGC 315 turns out to be quite gas rich, the total amount of H I within $400 \mathrm{kpc}$ of NGC 315 is $2.7 \times 10^{9} M_{\odot}$. It is clear that NGC 315 is the dominant galaxy of the group. In such an environment it is not unlikely that a small, gas-rich companion has fallen into NGC 315.

\section{VLBI observations}

Earlier VLBI H I observations of NGC 315 have been presented by Peck (1999). However, in these observations the observing 

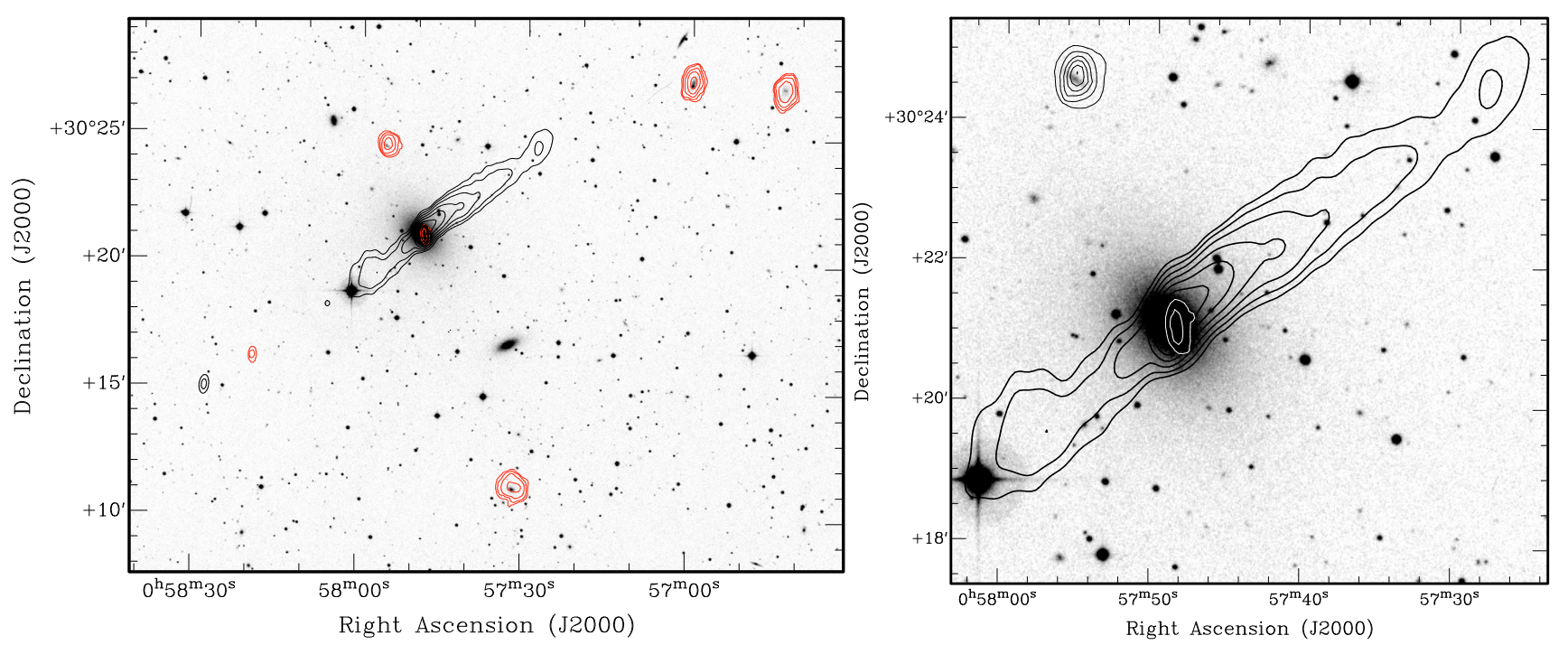

Fig. 4. Left: Contours (red) of the integrated H I emission derived from the WSRT observations, on top of an optical image obtained from the DSS. Contour levels are 4, 8, 16 and $32 \times 10^{19} \mathrm{~cm}^{-2}$. Also given are the contours (black) representing the continuum image derived from the same data. Contour levels of the continuum are 5, 10, 20,40, . mJy beam ${ }^{-1}$. Right: Detail of the figure on the left, showing the H I emission detected in NGC 315 and one nearby companion (thin contours) as well as the continuum (thick contours). Same contour levels as on the left.

Table 2. H I emission from other galaxies in the field of NGC 315.

\begin{tabular}{lccccc}
\hline \hline Name & \multicolumn{2}{c}{ Position $(\mathrm{J} 2000)$} & $\begin{array}{c}V_{\text {sys }} \\
\left(\mathrm{km} \mathrm{s}^{-1}\right)\end{array}$ & $\begin{array}{c}M_{\mathrm{HI}} \\
\left(10^{7} M_{\odot}\right)\end{array}$ & $\begin{array}{c}\text { sep } \\
(\mathrm{kpc})\end{array}$ \\
\cline { 2 - 3 } & $\mathrm{RA}$ & Dec & 4888 & 66.7 & 214 \\
$\mathrm{~J} 05730+3011$ & $00^{\mathrm{h}} 57^{\mathrm{m}} 30^{\mathrm{s}}$ & $30^{\circ} 11^{\prime} 05^{\prime \prime}$ & 4280 & 4.0 & 164 \\
$\mathrm{~J} 05819+3016$ & $00^{\mathrm{h}} 58^{\mathrm{m}} 19^{\mathrm{s}}$ & $30^{\circ} 16^{\prime} 05^{\prime \prime}$ & 5280 & 75.2 & 75 \\
$\mathrm{~J} 05755+3024$ & $00^{\mathrm{h}} 57^{\mathrm{m}} 55^{\mathrm{s}}$ & $30^{\circ} 24^{\prime} 32^{\prime \prime}$ & 4208 & 38.2 & 308 \\
J05642+3027 & $00^{\mathrm{h}} 56^{\mathrm{m}} 42^{\mathrm{s}}$ & $30^{\circ} 27^{\prime} 13^{\prime \prime}$ & 5007 & 77.8 & 307 \\
J05700+3027 & $00^{\mathrm{h}} 56^{\mathrm{m}} 59^{\mathrm{s}}$ & $30^{\circ} 27^{\prime} 15^{\prime \prime}$ & 5246 & 83.4 & 246 \\
\hline
\end{tabular}

The last column (sep) gives the distance (in kpc) of these galaxies from NGC 315.

band was centered on the velocity of the narrow $\mathrm{H}$ I absorption, as the presence of a broad absorption component was not yet established at the time. The new VLBI observations presented here use a broader bandwidth and a central frequency that allows us to detect both H I components.

NGC 315 was observed with the Global VLBI Network in November 2001. We used a bandwidth of $8 \mathrm{MHz}$ in 512 channels. The velocity resolution, after Hanning smoothing, is $\sim 6 \mathrm{~km} \mathrm{~s}^{-1}$. The rms noise is $\sim 0.26 \mathrm{mJy}^{\text {beam }}{ }^{-1}$ in the continuum image and $\sim 0.9 \mathrm{mJy} \mathrm{beam}^{-1}$ in the line channels.

The continuum image obtained from the line-free channels (beam $9 \times 3$ mas p.a. $=-20^{\circ}$ using natural weighting) is shown in Fig. 2. A strong core and a jet are detected, consistent with previous observations (see e.g. the detailed observations presented in Cotton et al. 1999). The position angle of the jet is about $43^{\circ}$, consistent with that of the large-scale jet and perpendicular to dust disk observed by HST (see Fig. 1). The peak of the continuum is about $190 \mathrm{mJy}^{\text {beam }}{ }^{-1}$. In the following sections we present the results obtained from the VLBI line data.

\section{The $\mathrm{H} I$ absorption at pc-scale and the origin of the atomic neutral hydrogen}

Both the narrow and the broad H I absorption systems are detected and spatially resolved by the VLBI observations. As we discuss below, both the kinematics and the column density distribution are quite different for the two components, suggesting that the two absorption systems have very distinct origins.

\subsection{The broad $\mathrm{HI}$ absorption}

An interesting but puzzling result from the VLBI observations is the morphology and kinematics of the broad H I absorption (Figs. 5 left and 6). Some absorption is detected against the core, but there is a strong gradient in column density, the column density being much higher further down the jet. Together with the density gradient, there is also a velocity gradient along the jet, with the gas further away from the nucleus having a larger redshift. The derived column density has a peak of $\sim 1 \times 10^{19} T_{\text {spin }}$ $\mathrm{cm}^{-2} \mathrm{~K}^{-1}$ (where the filling factor $f$ has been taken as 1 given that the absorption is resolved), while the column density in front of the nucleus is about a factor 10 lower. Further down the jet, no absorption is detected, but this is most likely due to the limited sensitivity of the VLBI observations. Indeed, about $30 \%$ of the flux detected for the broad absorption in the WSRT and VLA observations is missing in the VLBI observations.

One can attempt to make a rough estimate of the mass of the H I producing the broader absorption. Taking an average column density of $2 \times 10^{20} \mathrm{~cm}^{-2}$ (for a canonical $T_{\text {spin }}=100 \mathrm{~K}$ ) and an area of $10 \times 3$ parsec (corresponding to $30 \times 10$ mas), the mass enclosed would be about $100 M_{\odot}$. This number is most likely a lower limit because of a number of uncertain parameters. Most uncertain is perhaps the size of the gas cloud: part of the flux is missing and, therefore, the absorption is likely to be more extended than can be recovered from VLBI observations. In addition, of course, the $\mathrm{H}$ I detected is only that part of the gas cloud that is in front of the jet. Moreover, the assumed value for 

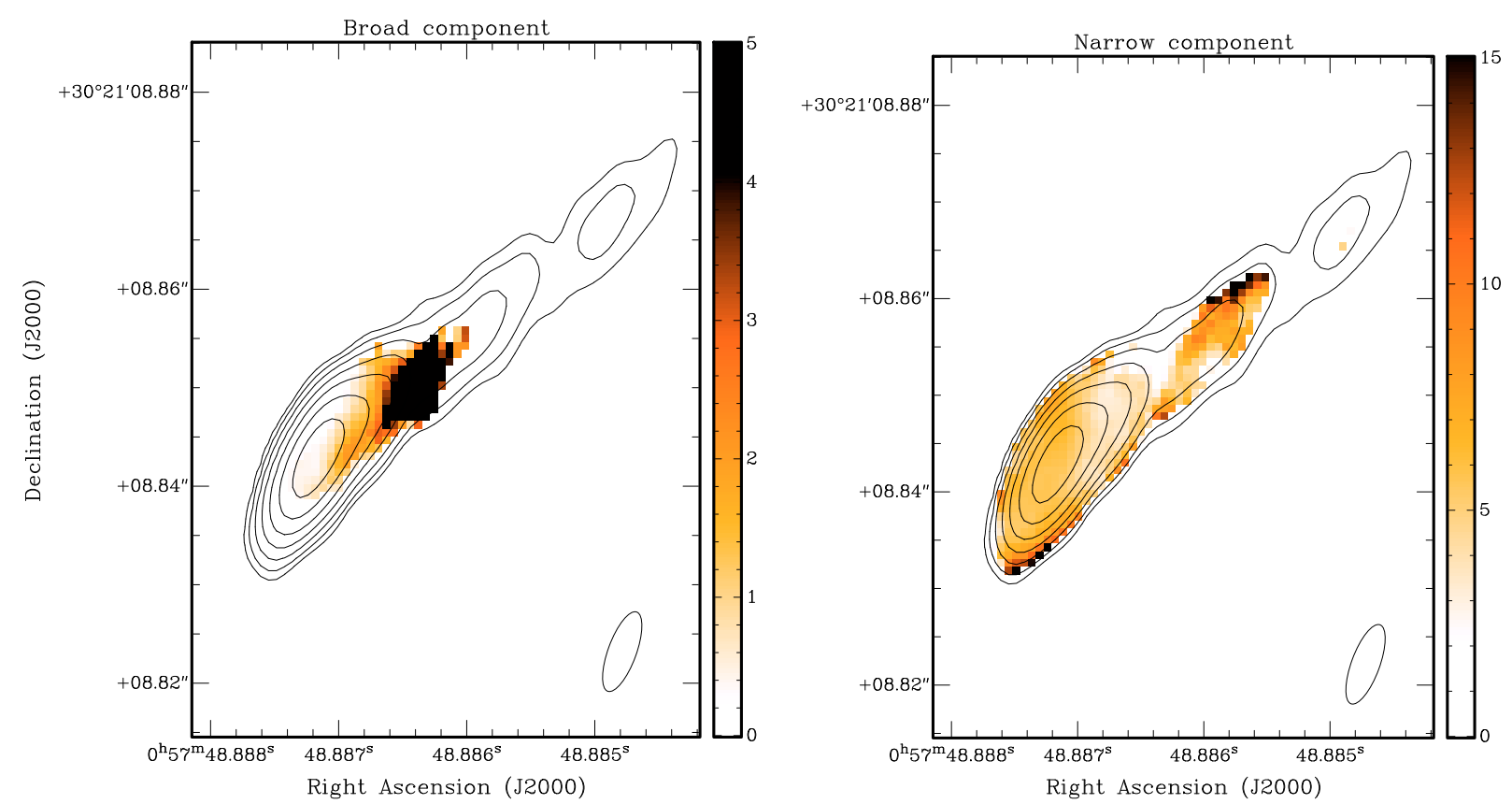

Fig. 5. Continuum (contours) and column density (grayscale) of the broad (left) and narrow (right) H I absorption as derived from the VLBI observations. The intensity scales given on the right side of each panel are in units of $10^{20} \mathrm{~cm}^{-2}$. The contours of the continuum are $2,4,8,16, \ldots$ mJy beam ${ }^{-1}$.

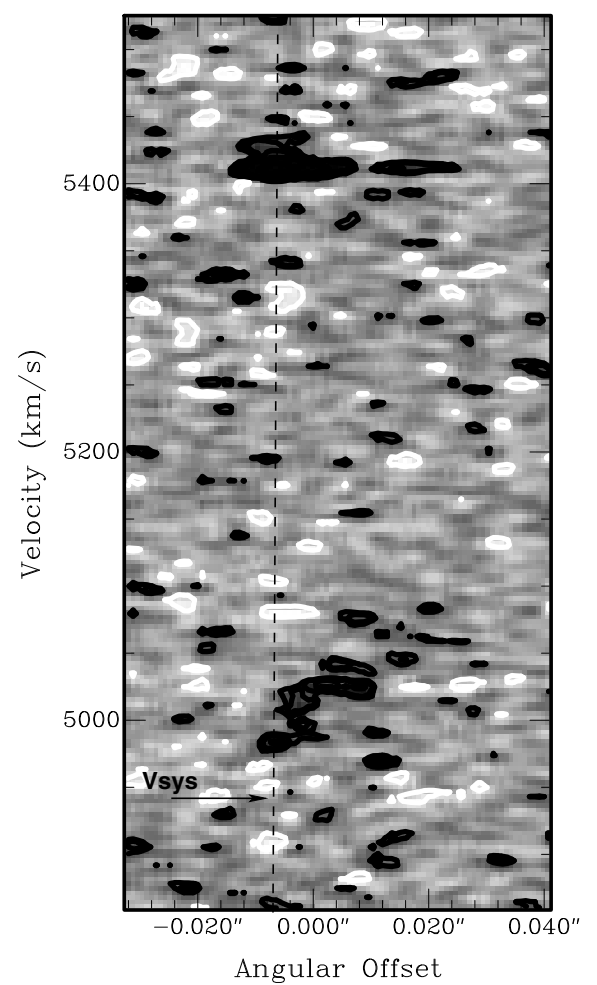

Fig. 6. Position-velocity plot taken along the radio jet (p.a. $\sim 130^{\circ}$ ). The plot shows both absorption features, centered around 5000 and $5400 \mathrm{~km} \mathrm{~s}^{-1}$, respectively. The velocity gradient in the H I broader component is visible. The contour levels are $-0.0019,-0.0025,-0.0038$, $-0.01,-0.03,0.0019 \mathrm{mJy}^{-1}$ beam $^{-1}$. Negative contours (representing the absorption) are in black. The position of the core and the systemic velocity are indicated.

the $T_{\text {spin }}$ is uncertain. The gas producing the $\mathrm{H}$ I absorption may be located very close to the nucleus (see next section) and, therefore, the assumption of $T_{\text {spin }}=100 \mathrm{~K}$ could be unrealistic. The
$T_{\text {spin }}$ might well be at least a factor 10 higher (see also results on PKS 1549-79 by Holt et al. 2006). X-ray observations suggest that the AGN is seen through a medium with a column density of $5 \times 10^{21} \mathrm{~cm}^{-2}$ (Worrall et al. 2003), indeed suggesting that the spin temperature is substantially higher than $100 \mathrm{~K}$. Therefore, $1000 M_{\odot}$ is a more likely lower limit to the H I mass. In this context, it is quite conceivable that the observed gradient in column density more reflects a gradient in $T_{\text {spin }}$ and not a gradient in the actual density.

\subsection{Nature of the cloud producing the broad $\mathrm{HI}$ absorption}

The strong gradients in the H I column density and velocity of the broad absorption suggest that the gas is being influenced by the presence of the AGN, indicating that this gas is physically close to the core of NGC315. Moreover, as has been noted about broad $\mathrm{H}$ I absorption in other objects (see for example the case of Cygnus A described in Conway \& Blanco 1995), a major problem of broad absorption being due to a chance superposition of a foreground cloud (e.g. similar to the dust patches SW of the core seen in the optical) is the large velocity width and gradient of the broad H I absorption. For clouds in the ISM of galaxies, even if one considers clouds that are "wreckage" of some accretion event, one would expect to observe velocity gradients much smaller than the $100 \mathrm{~km} \mathrm{~s}^{-1}$ over only $7 \mathrm{pc}$ observed for the broad absorption.

However, although it is likely that the broad absorption occurs close to the AGN, the nature of the gas resulting in the broad absorption is not clear. One interpretation which may fit the data is that of a circumnuclear torus. A configuration in which we are looking through a section of a thick rotating torus comprised mainly of atomic gas would result in a broad line width. In cases where the radio source axis is in the plane of the sky and our line of sight passes through the midplane of the torus, even broader lines have been found, and in almost all cases detected to date, absorption features attributable to a circumnuclear torus are redshifted with respect to the systemic velocity of the host 
galaxy (e.g. PKS 2322-123, FWHM $\sim 700 \mathrm{~km} \mathrm{~s}^{-1}$ redshifted by $\sim 220 \mathrm{~km} \mathrm{~s}^{-1}$; Taylor et al. 1999). This is probably due to streaming motions within the structure toward the accretion disk and central supermassive black hole.

In a source which has a jet axis close to the plane of the sky, we would expect to see absorption from a torus toward the inner jets on both sides of the core (e.g. Peck et al. 1999), with a smaller optical depth possible toward the core due to much higher spin temperatures near the AGN, as described above. However in a radio source which is oriented more toward our line of sight, such as NGC 315, the counterjet is Dopplerdimmed, and the plane of the disk is not aligned with our vantage point. Thus we do not necessarily expect to have the sensitivity to detect absorption toward the inner counterjet, as the continuum source is very faint, and we are looking along a line of sight from the top through the middle of the torus, where much of the gas may lie in the region of higher spin temperature and thus yield a lower optical depth.

Where the circumnuclear torus model struggles in this source, however, is in explaining the compact region of higher column density shown in Fig. 5. Some circumnuclear tori have been shown to be clumpy on similar scales (Peck \& Taylor 2001), but not with this range of density. Additionally, it would be hard to explain the velocity gradient along the jet unless this might be due to the gas above the midplane of the torus streaming down and inward toward the central mass. Such a scenario would result in an inward component of the velocity vector which would point more directly away from our vantage point as one went higher in scale height, yielding a larger redshift. The gas further down the jet would also have a component of inward streaming motion in addition to rotation, but given the orientation of the torus, these motions would not be directly away from us, and thus would not give rise to as large a redshift. Alternatively, one might explain both the compact region of high column density and the cospatial region of higher redshift with a discrete cloud falling toward a circumnuclear structure, but we do not have the spatial or velocity resolution to justify this level of detail in the model. Thus at present, we cannot rule out the possibility of a circumnuclear toroidal structure, but more corroborating data would be required before we could adopt this model.

Another possibility is that the broad absorption is due to gas entrained in the jet. NGC 315 does contain a number of small gas/dust clouds (see below), so it is, in principle, possible that one of these is now interacting with the jet. A structure of (molecular) gas aligned with radio jet has been found in M51 (Matsushida, Muller, \& Lin 2007). Also in that object, a velocity gradient with blueshifted velocities closer to the nucleus has been observed. The authors argue that the observed structure and velocity gradient are due to molecular gas entrained by the radio jet. Naively, one would however expect that if gas is entrained in a jet, the velocities would appear more blueshifted (i.e. coming toward the observer dragged by the jet) as one goes along the jet, opposite to these observations. We note in passing that the broad absorption is redshifted with respect to the systemic velocity, but that it is blueshifted relative to the narrow absorption. If the velocity of the narrow absorption is representative for small clouds falling into NGC 315, the jet would then be pushing the gas out with (projected) blueshifts of about $400 \mathrm{~km} \mathrm{~s}^{-1}$. One complication is that it would be have to be a coincidence that the velocity of the outflowing gas ends up to be close to the systemic velocity of the galaxy. It would also still not explain the velocity gradient along the jet.
Finally, let us consider the possibility that the broad absorption is somehow related to the process of providing (directly or indirectly) material for fueling the activity in the nucleus, i.e. gas (not necessarily part of a circumnuclear structure) that is falling into the nucleus. One can estimate, albeit with considerable uncertainty, the mass accretion rate that would be associated to this process, simply from the $\mathrm{H}$ I mass and the timescale for falling in. For these calculations it is, however, important to keep in mind (as described in details above) that the HI detected in absorption may be just a small fraction of the HI present around the nucleus. This is because we are limited to the gas in front of the radio continuum. This means that the numbers derived here (in particular the accretion rate) can be quite conservative lower limits. Taking $50 \mathrm{~km} \mathrm{~s}^{-1}$ as the infalling velocity of the gas, the timescale would be $\sim 7 \times 10^{5} \mathrm{yr}$. This would give accretion rates of at least $10^{-4} M_{\odot} \mathrm{yr}^{-1}$ and more likely $10^{-3} M_{\odot} \mathrm{yr}^{-1}$, if we adopt the mass of the gas estimated above with the assumption for $T_{\text {spin }}=3000 \mathrm{~K}$. An accretion rate of $10^{-3} M_{\odot} \mathrm{yr}^{-1}$ is very similar to those derived for other X-ray luminous elliptical galaxies, based on Chandra observations (Allen et al. 2006). On the other hand, using the luminosity of the radio core, one can estimate the power of the jet and from that the accretion rate (see Balmaverde et al. 2008). Starting from a radio core flux of $210 \mathrm{mJy}$, we find $P_{\text {core }} \sim 10^{30} \mathrm{erg} \mathrm{s}^{-1} \mathrm{~Hz}^{-1}$. Using the relationship between core luminosity and jet power (Heinz et al. 2007) this corresponds to $\log P_{\text {jet }} \sim 44.15 \mathrm{erg} \mathrm{s}^{-1} \mathrm{~Hz}^{-1}$. This can be converted in an accretion power of $\log P_{\text {accretion }} \sim 45.8 \mathrm{erg} \mathrm{s}^{-1} \mathrm{~Hz}^{-1}$ (Balmaverde et al. 2008) implying a mass accretion rate $\dot{M}_{\odot} \sim 0.1 M_{\odot} \mathrm{yr}^{-1}$. This accretion rate is quite high when compared to e.g. the sample in Allen et al. (2006). This high feeding rate is consistent with the high core power in NGC 315 relative to the galaxies in that sample. This would suggest that in the case of NGC 315, the hot gas dominates the accretion, unless the physical size of the cloud responsible for the broad absorption is much larger (a factor 100) than what we have assumed.

It is worth mentioning that in the case of the powerful radio galaxy Cygnus A, a cloud of molecular gas has been detected along the radio jet. This cloud (that has a velocity redshifted compared to the systemic velocity of the host galaxy) has been interpreted as falling into the nucleus and perhaps connected to fueling the AGN (Bellamy \& Tadhunter 2004). These authors argue that the connection may also be indirect, with the process of capturing the cloud and the subsequent settling into the circumnuclear disk leading to radial motions in the disk and increased fueling rate. The tentative detection of an $\mathrm{H} \mathrm{I}$ counterpart to this cloud in Cygnus A (Morganti et al. in preparation) makes the similarity to NGC 315 more evident.

\subsection{The narrow $\mathrm{HI}$ absorption}

Like the broad absorption, the narrow H I absorption is also spatially resolved in the VLBI observations. This absorption component covers about 20 mas of the source, $\sim 9 \mathrm{pc}$, from the core to the first part of the jet (see Fig. 5 right). The narrow absorption is different from the broad one in all respects. The column density distribution is much more uniform, the velocity is much narrower and no velocity gradient is observed. The fact that, contrary to what is seen for the broad absorption, the properties of the narrow absorption do not show any relation with the location of the AGN, or gradient along the jet, suggests that the cloud producing the narrow absorption is not physically close to the AGN. The very narrow velocity width also supports this (see also Dressel 1983). Moreover, the narrow absorption is close in velocity (and perhaps also in space, i.e. at large radii) to the $\mathrm{H} \mathrm{I}$ 
emission detected in the WSRT observation. This suggests that the narrow absorption is due to a cloud similar to those seen in optical images as absorbing clouds out to several kpc SW of the nucleus (Fig. 1). Using HST data, de Ruiter et al. (2002) derive a typical gas column density of $2.7 \times 10^{20} \mathrm{~cm}^{-2}$ for the gas clouds seen in NGC 315 , a value very similar to that found in our radio observations for the $\mathrm{H} \mathrm{I}$ in emission. We obtain the same column density for the narrow absorption if we assume $T_{\text {spin }}=100 \mathrm{~K}$. This could further support the idea that the gas is at larger radius since such value for $T_{\text {spin }}$ is characteristic for the ISM not affected by the active nucleus. If these clouds are the result of a small accretion event, one would indeed expect a redshifted velocity (Dressel 1983). Our low-resolution observations have shown that the environment of NGC 315 contains several small, gas-rich galaxies, so accretion of a small companion by NGC 315 is likely to have happened.

There are only two other cases known of radio galaxies where two such distinct HI absorbing systems have been observed. NGC 1275 (3C 84, van Gorkom et al. 1989 and references therein) and 4C 31.04 (Mirabel 1990). In both cases, a redshifted narrow component has been observed in addition to a broad closer to the systemic. For both objects, the redshifted absorption has been explained as being due to material falling into the main galaxy and it appears that the same explanation also applies to NGC 315.

\section{Summary}

We have studied the properties of the $\mathrm{HI}$ in the radio galaxy NGC 315. Two H I absorption components are present, a broad one $\left(\right.$ FWZI $\left.\sim 150 \mathrm{~km} \mathrm{~s}^{-1}\right)$ redshifted $\left(\sim 80 \mathrm{~km} \mathrm{~s}^{-1}\right)$ with respect to the systemic velocity, and a very narrow component (FWZI $\sim 8 \mathrm{~km} \mathrm{~s}^{-1}$ ). Interestingly, the two absorption components have very different properties. We also detect $\mathrm{H}$ I emission in NGC 315, a few kpc SW of the nucleus. This emission is likely associated with absorption patches that are observed in optical images.

The broad absorption shows a strong gradient in column density (or spin temperature) along the jet, with the highest densities (or lowest spin temperatures) furthest away from the AGN. It also shows a strong velocity gradient (more than $100 \mathrm{~km} \mathrm{~s}^{-1}$ over $10 \mathrm{pc}$ ) with the more redshifted velocities away from the AGN. The properties of this broad component strongly suggest that the gas producing the absorption is physically close to the AGN. The possibility that it is entrained by the radio jet (and partly responsible of the deceleration of the jet) appears unlikely because of the redshifted velocities of the gas. Gas located in a thick circumnuclear toroidal structure, with orientation similar to the dusty, circumnuclear disk observed with HST, cannot be completely ruled out although it appears difficult to reconcile with the observed morphology of the absorption and it would require inward streaming motion in addition to rotation. The scenario we favor is that the gas producing the broad absorption could be (directly or indirectly) connected with the fueling of the AGN, i.e. gas that is falling into the nucleus. If this is the case, the accretion rate derived is similar (considering all uncertainties) to that found for other X-ray luminous elliptical galaxies, although lower than that derived from the radio core luminosity for NGC 315 .

On the other hand, the properties of the narrow absorption are very uniform. Moreover, it tightly connects, in space and in velocity, with the H I emission in NGC 315. Most likely, the cloud responsible for the narrow absorption is quite far from the AGN and is likely due to material falling into NGC 315 . Five nearby, small gas-rich companions are also detected in $\mathrm{H}$ I. This implies that the environment of NGC 315 is quite gas rich and that accretion of gas from the environment is quite likely.

Acknowledgements. We would like to acknowledge Natascha Boric. Part of this work was done during her ASTRON/JIVE Summer Student project 2002. We would like to thanks Jesús González for further inspecting the optical spectral of NGC 315 and provide us the most accurate value of the systemic velocity. The Westerbork Synthesis Radio telescope is operated by the ASTRON (Netherlands Institute for Radio Astronomy) with support of the Netherlands Foundation for Scientific Research (NWO). The National Radio Astronomy Observatory is a facility of the National Science Foundation operated under cooperative agreement by Associated Universities, Inc. This research has made use of the NASA Extragalactic Database (NED), whose contributions to this paper are gratefully acknowledged. The Digitized Sky Survey was produced at the Space Telescope Science Institute under US Government grant NAG W-2166. The European VLBI Network is a joint facility of European, Chinese, South Africa, and other radio institutes funded by their national research councils.

\section{References}

Allen, S. W., Dunn, R. J. H., Fabian, A. C., Taylor, G. B., \& Reynolds, C. S. 2006, MNRAS, 372, 21

Antonucci, R. 1993, ARA\&A, 31, 473

Balmaverde, B., Capetti, A., \& Grandi, P. 2006, A\&A, 451, 35

Balmaverde, B., Baldi, R. D., \& Capetti, A. 2008, A\&A, 486, 119

Bellamy, M. J., \& Tadhunter, C. N. 2004, MNRAS, 353, 105

Best, P. N., et al. 2005, MNRAS, 362, 25

Best, P. N., et al. 2007, New Astron. Rev., 51, 168

Bridle, A. H., Davis, M. M., Fomalont, E. B., Willis, A. G., \& Strom, R. G. 1979, ApJ, 228, L9

Briggs, D. 1995, Ph.D. Thesis, New Mexico Inst. Mining Tech.

Butcher, H. R., van Breugel, W., \& Miley, G. 1980, ApJ, 235, 749

Canvin, J. R., Laing, R. A., Bridle, A. H., \& Cotton, W. D. 2005, MNRAS, 363 , 1223

Capetti, A., de Ruiter, H. R., Fanti, R., et al. 2000, A\&A, 362, 871

Capetti, A., Celotti, A., Chiaberge, M., et al. 2002, A\&A, 383, 104

Chamaraux, P., Balkowski, C., \& Fontanelli, P. 1987, A\&AS, 69, 263

Chiaberge, M., Capetti, A., \& Celotti, A. 1999, A\&A, 349, 77

Chiaberge, M., Macchetto, D. F., Sparks, W. B., et al. 2002, ApJ, 571, 247

Conway, J. 1996, in Extragalactic Radio Sources, ed. Ron D. Ekers, C. Fanti, \&

L. Padrielli (Kluwer Academic Publishers), IAU 175, 92

Conway, J. 1999, New Astron. Rev., 43, 509

Conway, J. E., \& Blanco, P. R. 1995, ApJ, 449, 131

Cotton, W. D., Feretti, L., Giovannini, G., Lara, L., \& Venturi, T. 1999, ApJ, 519, 108

Croton, D., et al. 2006, MNRAS, 365, 11

de Ruiter, et al. 2002, A\&A, 396, 857

de Young, D., Roberts, M. S., \& Saslaw, W. C. 1973, ApJ, 185, 809

Dressel, L. L., Davis, M. M., \& Bania, T. M. 1983, ApJ, 266, L97

Emonts, B .H. C. 2006, Ph.D. Thesis, University of Groningen

Emonts, B .H. C., Morganti, R., Tadhunter, C. N., et al. 2006, A\&A, 454, 125

Forbes, D. A. 1991, MNRAS, 249, 779

Garcia, A. M. 1993, A\&A Suppl. Ser., 100, 47

Gupta, N., \& Saikia, D. J. 2006, MNRAS, 370, 738

Heckman, T. M., Balick, B., van Breugel, W. J. M., \& Miley, G. K. 1983, AJ, 88, 583

Heinz, S., Merloni, A., \& Schwab, J. 2007, ApJ, 658, L9

Holt, J., Tadhunter, C., Morganti, R., et al. 2006, MNRAS, 370, 1633

Holt, J., Tadhunter, C. N., \& Morganti, R. 2008, MNRAS, 387, 639

Laing, R. A., Canvin, J. R., Cotton, W. D., \& Bridle, A. H. 2006, MNRAS, 368, 48

Leon, S., Lim, J., Combes, F., \& Dihn-V-Trung. 2003, in Active Galactic Nuclei: from Central Engine to Host Galaxy, ed. S. Collin, F. Combes, \& I. Shlosman, ASP Conf. Ser., 290, 525

Knapp, G. R., Bies, W. E., \& van Gorkom, J. H. 1990, AJ, 99, 476

Mack, K.-H., Klein, U., O’Dea, C. P., Willis, A. G., \& Saripalli, L. 1998, A\&A, 329,431

Matsumoto, Y., Fukazawa, K., Iyomoto, N., \& Makishima, K. 2001, Publ. Astr. Soc. Jpn., 53, 475

Mirabel, I. F. 1990, ApJ, 352, L37

Morganti, R. 2002, in Issues in Unification of AGNs, ed. R. Maiolino, A. Marconi, \& N. Nagar, ASP Conf. Ser., 258, 63 [arXiv:astro-ph/ Q109056]

Morganti, R., Oosterloo, T. A., Tadhunter, C. N., et al. 2001, MNRAS, 323, 331

Morganti, R., Oosterloo, T. A., Tadhunter, C. N., et al. 2004, A\&A, 424, 119 
Morganti, R., Tadhunter, C. N., \& Oosterloo, T. A. 2005, A\&A, 444, L9 Morganti, R., Emonts, B., Holt, J., et al. 2009, AN, 330, 233

Noel-Storr, J., Baum, S. A., Verdoes Kleijn, G., et al. 2003, ApJS, 148, 419

Peck, A. B. 1999, Ph.D. Thesis, New Mexico Inst. of Mining and Technology Peck, A. B., \& Taylor, G. B. 2001, ApJ, 554, L14

Peck, A. B., Taylor, G. B., \& Conway, J. E. 1999, ApJ, 521, 103

Salomé P., et al. 2006, A\&A, 454, 437

Sault, R. J., Teuben, P. J., \& Wright, M. C. H. 1995, in Astronomical Data Analysis Software and Systems IV, ed. R. Shaw, H. E. Payne, \& J. J. E. Haynes, ASP Conf. Ser., 77, 433

Shostak, G. S., van Gorkom, J. H., Ekers, R. D., et al. 1983, A\&A, 119, L3
Taylor, G. B., O’Dea, C. P., Peck, A. B., \& Koekemoer, A. M. 1999, ApJ, 512, L27

Trager, S. C., Faber, S. M., Worthey, G., \& Jesús González, J. 2000, AJ, 119, 1645

van Gorkom, J. H., Knapp, G. R., Ekers, R. D., et al. 1989, AJ, 97, 708

Vermeulen, R., et al. 2003, A\&A, 404, 861

Wakker, B., Oosterloo, T. A., \& Putman, M. 2002, AJ, 123, 1953

Wilson, A. 1996, in Energy Transport in Radio Galaxies and Quasars, ed. Hardee, Bridle, Zensus, ASP Conf. Ser., 9

Worrall, D. M., Birkinshaw, M., \& Hardcastle, M. J. 2001, MNRAS, 326, L7 Worrall, D. M., Birkinshaw, M., \& Hardcastle, M. J. 2003, MNRAS, 343, L73 